\title{
A radar-based regional extreme rainfall analysis to derive the thresholds for a novel automatic alert system in Switzerland
}

\author{
Luca Panziera ${ }^{1,2,3}$, Marco Gabella ${ }^{1}$, Stefano Zanini ${ }^{1}$, Alessandro Hering ${ }^{1}$, Urs Germann ${ }^{1}$, and Alexis Berne ${ }^{2}$ \\ ${ }^{1}$ MeteoSwiss, Locarno Monti, Switzerland \\ ${ }^{2}$ Environmental Remote Sensing Laboratory, École Polytechnique Fédérale de Lausanne, Lausanne, Switzerland \\ ${ }^{3}$ Oeschger Centre for Climate Change Research, Institute of Geography, University of Bern, Bern, Switzerland \\ Correspondence to: Luca Panziera (luca.panziera@meteoswiss.ch)
}

Received: 21 March 2016 - Published in Hydrol. Earth Syst. Sci. Discuss.: 30 March 2016

Accepted: 26 May 2016 - Published: 15 June 2016

\begin{abstract}
This paper presents a regional extreme rainfall analysis based on 10 years of radar data for the 159 regions adopted for official natural hazard warnings in Switzerland. Moreover, a nowcasting tool aimed at issuing heavy precipitation regional alerts is introduced. The two topics are closely related, since the extreme rainfall analysis provides the thresholds used by the nowcasting system for the alerts. Warm and cold seasons' monthly maxima of several statistical quantities describing regional rainfall are fitted to a generalized extreme value distribution in order to derive the precipitation amounts corresponding to sub-annual return periods for durations of $1,3,6,12,24$ and $48 \mathrm{~h}$. It is shown that regional return levels exhibit a large spatial variability in Switzerland, and that their spatial distribution strongly depends on the duration of the aggregation period: for accumulations of $3 \mathrm{~h}$ and shorter, the largest return levels are found over the northerly alpine slopes, whereas for longer durations the southern Alps exhibit the largest values. The inner alpine chain shows the lowest values, in agreement with previous rainfall climatologies.

The nowcasting system presented here is aimed to issue heavy rainfall alerts for a large variety of end users, who are interested in different precipitation characteristics and regions, such as, for example, small urban areas, remote alpine catchments or administrative districts. The alerts are issued not only if the rainfall measured in the immediate past or forecast in the near future exceeds some predefined thresholds but also as soon as the sum of past and forecast precipitation is larger than threshold values. This precipitation total, in fact, has primary importance in applications for which
\end{abstract}

antecedent rainfall is as important as predicted one, such as urban floods early warning systems. The rainfall fields, the statistical quantity representing regional rainfall and the frequency of alerts issued in case of continuous threshold exceedance are some of the configurable parameters of the tool.

The analysis of the urban flood which occurred in the city of Schaffhausen in May 2013 suggests that this alert tool might have complementary skill with respect to radar-based thunderstorm nowcasting systems for storms which do not show a clear convective signature.

\section{Introduction}

\subsection{Rainfall monitoring, nowcasting and warning systems}

In order to increase preparedness and to reduce human and economic impacts of natural hazards caused by heavy precipitation, such as surface water flooding in urban areas, flash floods or debris flow, warnings are issued to local authorities and population when rainfall amounts exceed some threshold values over a given area in a certain period of time. The thresholds corresponding to the alert levels are usually given by the depth of observed rainfall for a given duration which is likely to cause floods according to examination of rainfall accumulations during previous flooding events, and they are often refined based on the findings of post-event analyses. Therefore, they tend to be specific to individual regions (e.g., Alfieri et al., 2012; Sene, 2013). Both rainfall depths 
and river discharges corresponding to predefined return periods are also used as thresholds for the alerts (e.g., Alfieri et al., 2011; Knechtl, 2013; Javelle et al., 2014; Fouchier et al., 2015). For some applications, precipitation thresholds depend also on antecedent rainfall: for example, when issuing landslide, debris flow or urban flooding warnings, the amount of precipitation measured in the hours or days preceding the onset of a storm event has to be carefully considered, since it strongly influences soil saturation conditions or the spare capacity in the drainage network of a city, playing a fundamental role in determining the severity of the hazard (e.g., Neary and Swift, 1987; Giannecchini et al., 2000; Wieczorek and Glade, 2005; Martina et al., 2006; Guzzetti et al., 2007; Sene, 2013). Soil moisture state is a basic input variable also of the US National Weather Service flash flood forecasting system. In such a tool, rainfall-runoff curves are computed on a regular basis for each basin taking into account current soil moisture conditions, and the average rainfall over a specified area and temporal interval required to initiate flooding on small streams (flash flood guidance) is thus obtained, once the threshold runoff is known (e.g., Sweeney, 1992).

Real-time automatic monitoring of precipitation and reliable rainfall forecasts are thus necessary ingredients to issue accurate and timely warnings, especially for flash floods, which occur rapidly and result in a limited opportunity for warnings to be prepared and issued (e.g., Collier, 2007). The large spatial and temporal variability of rainfall requires monitoring and forecasting systems capable of measuring and predicting precipitation with high spatial and temporal resolutions. Even though rain gauges provide precise rainfall measurements at the local scale, the operational ground station networks can rarely provide precipitation estimates with high spatial resolution: even in the European Alps, one of the regions with the densest rain gauge networks, typical spacing between stations is about $10 \mathrm{~km}$, whereas the precipitation distribution can vary at scales much smaller than $10 \mathrm{~km}$ (e.g., Frei and Schär, 1998; Germann and Joss, 2001; Isotta et al., 2014). Ground-based weather radars, on the other hand, can measure precipitation over large areas with high spatial and temporal resolution, even though the variability in the relation between reflectivity and rainfall intensity limits the accuracy of the measurements; moreover, the use of radar in mountainous regions requires proper solutions and corrections to the data (e.g., Germann et al., 2006). Combining rain gauges with radar measurements through geostatistical interpolation techniques is a valid solution to obtain reliable precipitation fields (e.g., Sideris et al., 2014a). Since the uncertainty of medium and long-range forecasts from numerical weather prediction models is still too large at the scale of individual storms and rainfall peaks, automatic warning systems usually need more accurate predictions with lead time shorter than $6 \mathrm{~h}$ (nowcasting) to issue reliable alerts for small geographical regions, such as urban areas or mountain catchments. Operational quantitative precipitation nowcasting is based on numerical weather prediction models and heuristic systems. Even though the former includes the full set of equations describing the atmospheric processes, the assimilation and the initialization cycles as well as the time required to obtain the forecast are still too long with respect to the needs of operational nowcasting (e.g., Panziera et al., 2011). On the other hand, nowcasting by radar-based heuristic systems, such as Lagrangian extrapolation or analogues, quickly provides forecasts of rainfall and outperforms numerical model forecasts for the first hours, even though it strongly suffers from the lack of growth and decay mechanisms able to predict the evolution of the storms for lead times longer than a few hours (e.g., Panziera et al., 2011; Mandapaka et al., 2012). Finally, some studies propose a merging of the forecasts obtained with numerical weather prediction models and heuristic systems (e.g., Golding, 1998; Bowler et al., 2006; Atencia et al., 2010; Haiden et al., 2011), but this approach will be successful as long as numerical models could provide good forecasts at the nowcasting spatiotemporal scales (Wilson et al., 2010).

\subsection{Radar-based extreme rainfall analysis}

Even though radar archives are nowadays a unique resource for investigating the behavior of precipitation, since weather radar has been widely used for quantitative precipitation estimation (QPE) for many years, radar data have not yet been extensively used to derive statistics of extreme rainfall. Among the first papers presenting extreme rainfall analyses based on radar QPE, Durrans et al. (2002) obtained depth-area ratios for several return periods for a large portion of western United States including part of Great Plains and the Rocky Mountains, by fitting a Gumbel distribution to annual radar rainfall maxima for durations of 1,2 and $4 \mathrm{~h}$ using a radar data set of 8 years. They state that the most significant limitations of radar-rainfall data, both for frequency analyses and for development of depth-area relationships, are the shortness of the archive and the heterogeneities caused by continual improvements in the data processing algorithms. In order to derive radar areal reduction factors, Allen and DeGaetano (2005) estimate areal precipitation depths for the 2-, 5- and 10-year return periods for New Jersey and North Carolina (United States) employing a 5 -year radar data set of daily rainfall. Overeem et al. (2009) employed 11 years of radar data adjusted using rain gauges to derive depth-duration-frequency curves for accumulation periods from $15 \mathrm{~min}$ to $24 \mathrm{~h}$ over the Netherlands. They also found reasonable agreement between the parameters of the Generalized Extreme Value (GEV) distribution derived with rain gauges and radar, showing that radar data are suitable to construct depth-duration-frequency curves. The potential of using radar QPE for rainfall-frequency analyses has been recently illustrated also by Marra and Morin (2015), who derived intensity-duration-frequency curves for durations of $20 \mathrm{~min}, 1$ and $4 \mathrm{~h}$ by using 23 years of radar data over Israel, a 
region characterized by steep climatic transitions. By means of a detailed comparison between gauge- and radar-derived IDF curves, they could show that weather radar is able to discern between climatic areas in terms of rainfall extremes and to identify extreme precipitation small-scale patterns in a region where regionalization approaches are very difficult to apply because of the strong rainfall gradients and sparse rain gauge stations.

\subsection{Objective of this paper}

The objective of this paper is two-fold: first, to present a radar-based regional extreme precipitation analysis for Switzerland; second, to introduce NowPAL (Nowcasting of Precipitation Accumulations), the nowcasting system recently developed at MeteoSwiss to issue precipitation alerts for predefined geographical regions. The two objectives are closely related, since the extreme rainfall analysis provides the rainfall thresholds used by NowPAL for the alerts. This article offers an intuitive and practical solution for both implementing an automatic regional rainfall alert system and for choosing the rainfall thresholds corresponding to the alert levels. The basic assumption is that in a country characterized by different precipitation regimes such as Switzerland (e.g., Isotta et al., 2014), an alert of a specific level should have the same probability to be issued in every region in a year. Thus, the alert thresholds should be the rainfall amounts corresponding to specific return periods. The originality of this article, with respect to previous works dedicated to extreme precipitation analysis, nowcasting and warning systems, lies in the following aspects.

1. The high spatial resolution of radar precipitation field is fully exploited, as rainfall maxima of several statistical quantities describing regional rainfall distribution are not only taken into account for the extreme value analysis but are also used by the nowcasting system to issue alerts.

2. The presented nowcasting system can be targeted for specific user requirements, as it is fully configurable and it is based on a simple and practical approach. Thus, it is appropriate to issue alerts for customers interested in specific applications and regions, such as small urban areas or alpine catchments.

3. The alerts are issued not only if the rainfall measured in the immediate past or forecast in the near future exceeds some predefined thresholds but also as soon as the sum of past and forecast precipitation is larger than threshold values. In fact, in operational nowcasting, the sum of accumulated and predicted rainfall is the quantity which actually drives the emergency decisions taken during heavy precipitation events (see Fig. 1).

4. Since the nowcasting system should issue alerts not only for rare events but also for frequent storms pro-

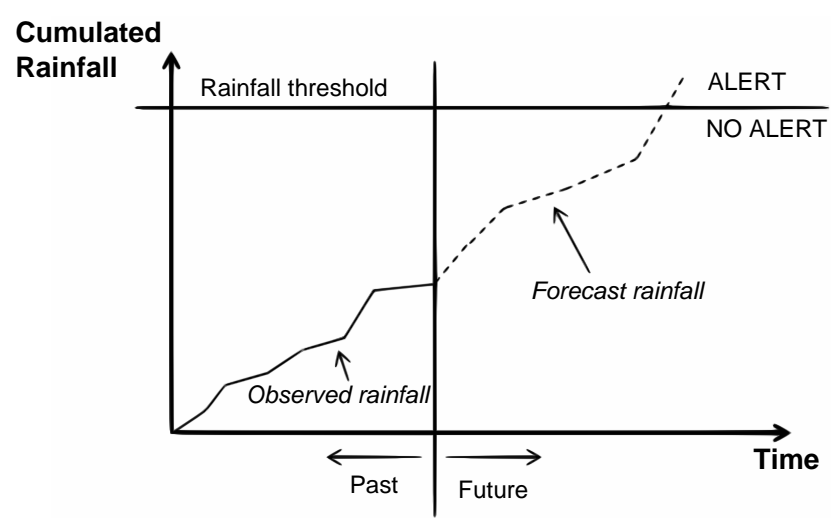

Figure 1. Schematic representation of threshold exceedance for a fixed accumulation period by considering the sum of observed and predicted precipitation. Adapted from Martina et al. (2006).

ducing rainfall amounts with sub-annual return periods, the analysis is performed taking into account monthly maxima of warm and cold seasons.

In this paper, the term alert indicates the situation in which the predefined rainfall thresholds are exceeded over a given region, and timely communications are fully automatically sent to the customers of the nowcasting system. Since the utility of the alerts and the performance of the system strongly depends on the quality of the ingested QPE products and on the skill of the forecasting systems, a verification of the alerts issued by NowPAL is beyond the scope of this work.

\subsection{Outline of this paper}

The data used by the NowPAL system to issue alerts in real time, as well as those employed for the statistical analysis, are described in Sect. 2, which also presents the methodologies adopted for the regional extreme rainfall analysis. The results of such analysis are presented in Sect. 3, whereas Sect. 4 illustrates the details of the NowPAL system. Section 5 provides an example of the functioning of the system through the analysis of an urban flood. Finally, Sect. 6 presents the main conclusions of this work.

\section{Data and methods}

This section introduces the data which are used in real time by the NowPAL system, together with the data set and the methodologies employed for the regional extreme rainfall analysis.

\subsection{Past rainfall}

Precipitation fields for past rainfall estimates are obtained from the MeteoSwiss operational radar product for QPE Germann et al. (2006), and from a recent radar-rain gauge merg- 
ing technique (Sideris et al., 2014a). Such products are used as QPE for both the real-time NowPAL system and the regional extreme value analysis.

The third-generation (3GEN) of Swiss radars, installed back in 1993 and consisting of three C-band Doppler radars, has been recently renewed. Two more radars were added to the network, in order to improve rainfall estimates in the inner alpine regions where the third-generation network had low visibility. Thus, the fourth-generation (4GEN) radar network is now composed by five C-band Doppler radars with state-of-the-art dual polarization technique (Germann et al., 2015). The operational scan strategy of the 3 GEN and 4GEN radars consists of 20 elevations between -0.2 and $40^{\circ}$ repeated every $5 \mathrm{~min}$. For the extreme value analysis presented in this paper, data from 2005 to 2015 were considered, excluding the year 2011, during which the 3GEN radars were substituted. The analysis was not extended further into the past since data prior to the year 2005 had only a global bias adjustment, while data from 2005 were adjusted for both local and global bias, giving more reliable precipitation measurements (Germann et al., 2006). Since the last 4GEN radar in Canton of Grisons is operational since the beginning of 2016, the rainfall maps used in this study are Cartesian composites derived from the measurements of three radars till 2013, four radars afterwards. The best radar estimate of precipitation at ground level is the result of sophisticated data processing based on more than 50 years of experience in radar operation in the alpine environment at MeteoSwiss (Joss and Lee, 1995; Germann et al., 2006). Such a product is retrieved through a weighted mean of all the volumetric radar observations above the ground, and radar data processing includes automatic hardware calibration, ground clutter elimination, visibility correction, correction for vertical profile of reflectivity, removal of residual nonweather echoes and bias correction (Germann and Joss, 2002; Germann et al., 2006). The latter compensates for systematic errors due to nonuniform beam filling, low-level growth not seen by the vertical profile correction and attenuation.

The merging of radar and rain gauge measurements is operationally performed at MeteoSwiss by CombiPrecip, a cokriging with external drift geostatistical method which incorporates both spatial and temporal information into the modelling and estimation technique Sideris et al. (2014a). CombiPrecip locally adjusts the radar rainfall map according to the values of the rain gauges; the main assumption is that the point rain gauge measurements are the primary, trustworthy data, while the radar data function as an external drift. The scheme includes a convection control routine, in order to reduce the negative effect on kriging of the scarce representativeness of rain gauge measurements in case of convective rainfall patterns Sideris et al. (2014b).

The horizontal spatial resolution of both radar and CombiPrecip precipitation maps is $1 \mathrm{~km} \times 1 \mathrm{~km}$. The temporal resolution of radar measurements is $5 \mathrm{~min}$, whereas CombiPrecip has a temporal resolution of $1 \mathrm{~h}$ before 2012, $5 \mathrm{~min}$ since
2013. For the statistical analysis presented here, 1 and $3 \mathrm{~h}$ rainfall accumulations were obtained from radar maps temporally aggregated every $5 \mathrm{~min}$, whereas $6 \mathrm{~h}$ and longer accumulations were obtained from CombiPrecip maps temporally aggregated every hour. The use of radar instead of CombiPrecip for short accumulations is motivated by the need to ingest these data as soon as possible in NowPAL and, in real time, radar maps are available before CombiPrecip. Moreover, using CombiPrecip to derive rainfall totals as short as 1 and $3 \mathrm{~h}$ would have led to large negative bias in precipitation totals corresponding to given return periods, since the temporal resolution of CombiPrecip available through the whole data set is $1 \mathrm{~h}$.

\section{Residual ground clutter removal}

The continuous repetition of low echoes due to residual ground clutter in radar QPE can produce isolated large rainfall totals over long temporal aggregations. For the study of extreme rainfall, therefore, the identification of radar measurements contaminated by ground clutter has primary importance. Even though ground clutter elimination algorithms are part of the sophisticated data processing of radar data, $3 \mathrm{GEN}$ radar rainfall estimates were still partially contaminated by clutter, in opposition to 4GEN radar data which are almost free from it. For this study, we employed the twosample Kolmogorov-Smirnov statistical test to identify the pixels significantly contaminated by residual ground clutter in 3GEN radar data. The two-sample Kolmogorov-Smirnov test is a nonparametric hypothesis test which can be used to state whether or not two samples are characterized by the same probability distribution (Kolmogorov, 1933; Smirnov, 1948). The test is based on the comparison between cumulative distribution functions $(C)$ of the two samples, the test statistic $D$ being the supremum of the set of their distances:

$D=\sup _{x}\left(\left|C_{1}(x)-C_{2}(x)\right|\right)$.

The null hypothesis assumes that two samples are described by the same probability distribution, and it is rejected if the test statistic is larger than a critical value which depends on the significance level $\alpha$. In our case, the two samples are the $5 \mathrm{~min}$ radar rainfall estimates at a given pixel for a year of 3GEN and a year of 4GEN data, and $\alpha$ was set to 0.01 . Assuming that the differences in distributions between 3GEN and 4GEN radar data due to residual ground clutter are more evident at low rainfall intensities, because of the frequent repetition of low intensity echoes, the range considered for the test was limited to $[0,5] \mathrm{mm} \mathrm{h}^{-1}$. Two pairs of years characterized by similar mean yearly precipitation in Switzerland were chosen: 2007 and 2013, 2008 and 2014. A Kolmogorov-Smirnov test was then applied to the two pairs of years, and the pixels which did not pass the test in both cases were considered affected by residual ground clutter and were not included in the extreme rainfall analysis. Fig- 
ure 3 shows the $3 \mathrm{~h}$ rainfall accumulation for three precipitation events which occurred between Italy and Switzerland, together with the resulting mask shaded in black. Such region is particularly affected by residual ground clutter, since the radar beam directly impacts high mountains towards the west as, for example, Monte Rosa (4634 m a.s.l.). Figure 3 shows that most of the cluttered pixels are located over high mountain peaks, where the presence of residual ground clutter could have effectively contaminated radar measurements.

We are aware that changes in the radar scan strategy from 3GEN to 4GEN radars might have led to the removal of noncluttered pixels, and that pixels affected by residual ground clutter in both $3 \mathrm{GEN}$ and $4 \mathrm{GEN}$ might not have been identified by this analysis. However, we assume that the results of this analysis are appropriate to the scope of this study.

\subsection{Future rainfall}

Information on rainfall expected in the near future is derived from three different products: COSMO, INCA and MAPLE. Such rainfall fields have not been used for the extreme value analysis, but they are used by the real-time NowPAL system.

COSMO (Consortium for Small-scale Modeling) is the numerical model operationally used at MeteoSwiss, as part of a major cooperative research effort between several national weather services in Europe (http://cosmo-model.org). It is a high-resolution, limited-area, nonhydrostatic numerical weather prediction model with the radar-rainfall observations assimilated using a latent heat nudging scheme. COSMO has a spatial resolution of $1 \mathrm{~km}$, forecasts are produced every $3 \mathrm{~h}$ and extend up to $33 \mathrm{~h}$ with $10 \mathrm{~min}$ temporal resolution.

INCA (Integrated Nowcasting through Comprehensive Analysis) is an operational nowcasting system producing a rainfall forecast based on Lagrangian extrapolation of the precipitation field for the first hour of forecast $(0-1 \mathrm{~h}$ lead times), and based on blending of extrapolation with COSMO forecasts from the second to the fourth hour (1-4h lead times); forecasts for longer lead times coincide with numerical model forecasts Haiden et al. (2011). The INCA precipitation analysis incorporates station data, radar data and elevation effects (orographic effects on rainfall). Forecasts are produced every $10 \mathrm{~min}$ as new radar and rain gauge measurements become available, and extend up to a $6 \mathrm{~h}$ lead time with 10 min temporal resolution.

MAPLE (McGill Algorithm for Precipitation Nowcasting) produces forecasts by first estimating the velocity field using variational echo tracking and then extrapolating the current radar image according to the velocity vectors previously derived (Germann and Zawadzki, 2002; Turner et al., 2004). This nowcasting system produces forecasts every $5 \mathrm{~min}$, up to a $12 \mathrm{~h}$ lead time with $5 \mathrm{~min}$ temporal resolution. Over the Alps, MAPLE has a larger skill with respect to COSMO forecasts for lead times shorter than $3 \mathrm{~h}$ (Mandapaka et al., 2012). At present, significant efforts are being made in order to in-

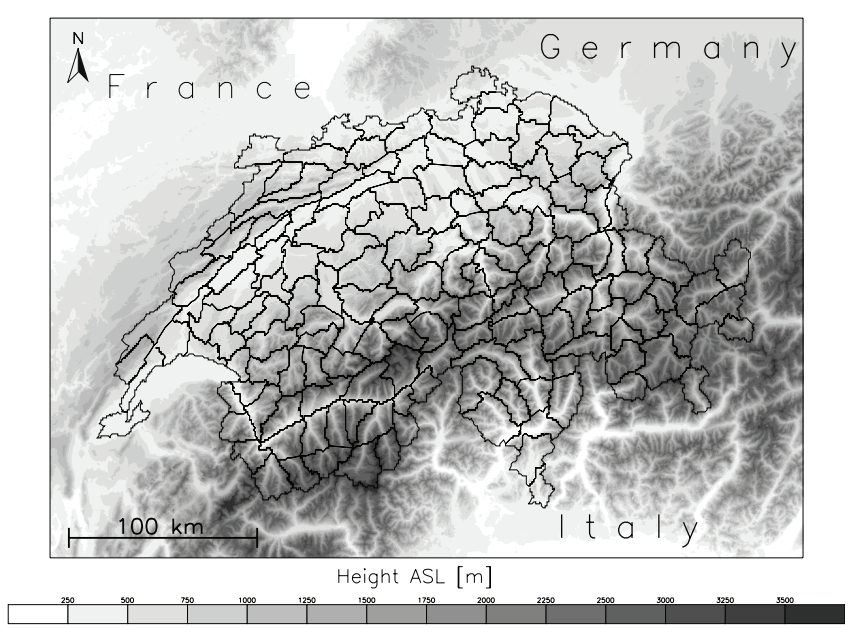

Figure 2. The 159 Swiss warning regions for which an extreme rainfall analysis is performed in this study.

clude the growth and decay processes due to the orographic forcing on the extrapolation scheme (Sideris et al., 2015).

\subsection{Extreme rainfall analysis}

The regional extreme rainfall analysis performed in this study has been conducted on radar and CombiPrecip precipitation fields aggregated over $N$ different temporal periods. In particular, radar data have been used to derive 1 and $3 \mathrm{~h}$ accumulations, whereas CombiPrecip product was aggregated to $6,12,24$ and $48 \mathrm{~h}$ (see Sect. 2.1). These temporal periods, which are called tot $t_{i}$ with $i=1, . ., 6$, have been selected because they are employed by the official MeteoSwiss warning system to issue alerts for the 159 Swiss warning regions shown in Fig. 2. The regions $\operatorname{reg}_{j}$, with $j=1, . ., 159$, were not defined as part of this study, since they are administrative districts or catchments; their area ranges from about 100 to $500 \mathrm{~km}^{2}$, with an average value of $264 \mathrm{~km}^{2}$. Different quantities describing the distribution of the temporally aggregated precipitation field within each warning region $R\left(\operatorname{tot}_{i}\right)_{j}$ were computed, exploiting the high spatial resolution of radar and CombiPrecip rainfall maps:

1. $\bar{R}\left(\operatorname{tot}_{i}\right)_{j}$, the average rainfall in period tot ${ }_{i}$ in the region $j$;

2. $\max \left(R\left(\operatorname{tot}_{i}\right)_{j}\right)$, the maximum of $1 \mathrm{~km}^{2}$ rainfall in period tot $_{i}$ in the region $j$;

3. $Q x\left(R\left(\operatorname{tot}_{i}\right)_{j}\right.$, the 90th percentile of rainfall in period tot $t_{i}$ in the region $j$; and

4. $\max \left(R S\left(\operatorname{tot}_{i}\right)_{j}\right)$, the maximum of regional rainfall in period tot ${ }_{i}$ in the region $j$ after a spatial aggregation of the original field with a square moving window of area $S \times S \mathrm{~km}^{2}$. 

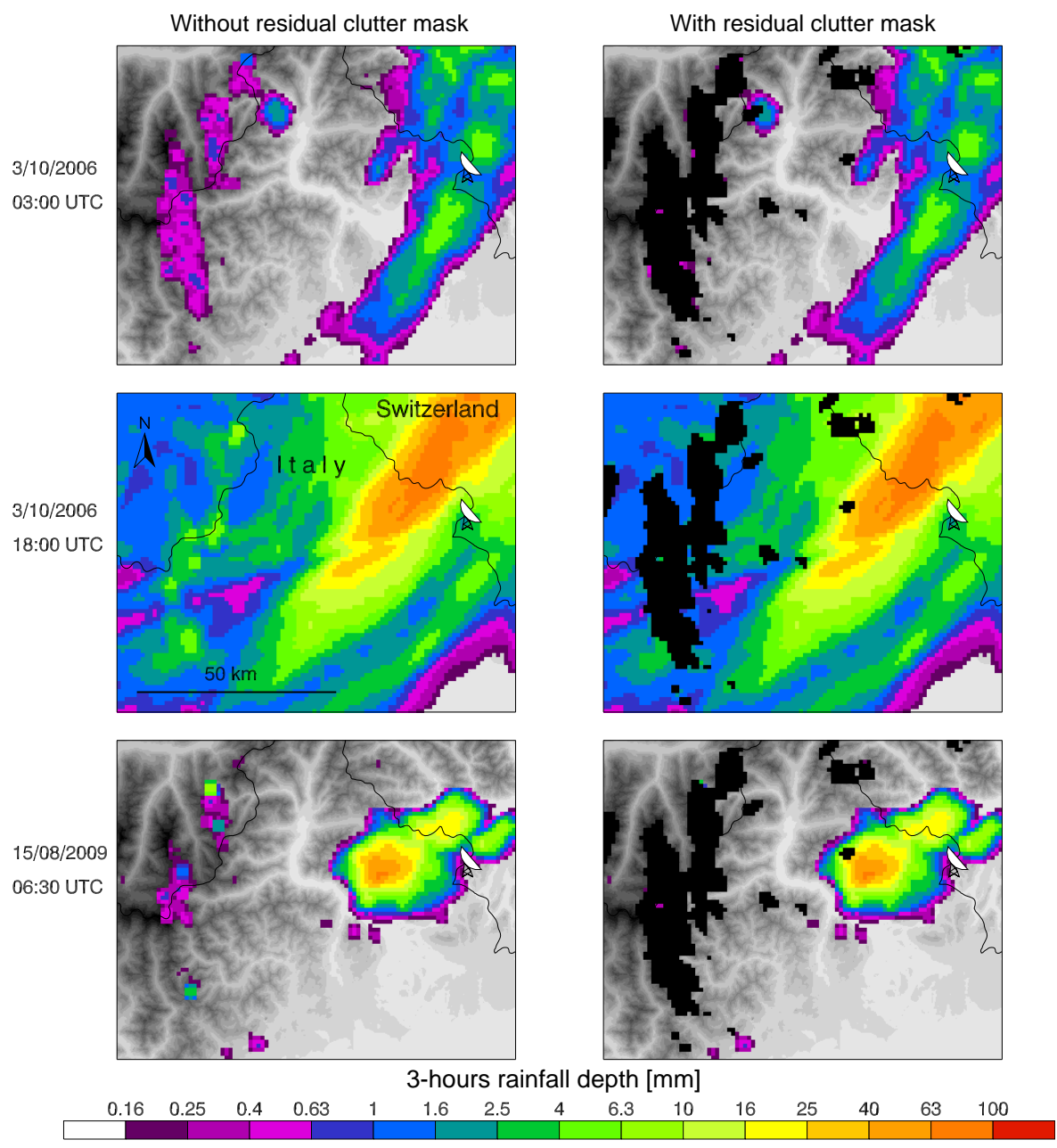

Figure 3. Zoom between Italy and Switzerland of the mask used to remove pixels affected by residual ground clutter in $3 \mathrm{GEN}$ radar data. The $3 \mathrm{~h}$ rainfall accumulations for three different time steps are shown in the left column, while the right column shows the same field with the pixels affected by residual ground clutter shaded in black. The location of the Monte Lema radar is indicated by the radar symbol.

These statistical quantities are called regional statistics and are indicated with $\operatorname{stat}\left(R\left(\operatorname{tot}_{i}\right)_{j}\right)$. Since regional statistics describe different aspects of regional rainfall distribution, they are required in order to provide a variety of solutions to NowPAL customers, which might be interested in different features of the rainfall field for a variety of applications. For example, while for hydrologic applications the average rainfall in the region is needed, the maximum rainfall in the region might be useful to emergency agencies which need to also take care of very localized, heavy rainfall. The aim of considering the maximum of precipitation after spatial aggregation with moving windows of a given area is motivated by the need to derive return periods of rainfall patterns of different sizes, so that an alert can be issued as soon as a rainfall pattern of that size is observed within the region, even though only a part of the region is affected by heavy rainfall.

The extreme value analysis presented here is based on a block maxima approach. Statistical modeling of the block maxima of precipitation is performed using the generalized extreme value (GEV) distribution (e.g., Coles, 2001) whose cumulative distribution function has the form:

$G(z)=\exp \left\{-\left[1+\xi\left(\frac{z-\mu}{\sigma}\right)\right]^{-\frac{1}{\xi}}\right\}$,

and it is defined on the set $\{z: 1+\xi(z-\mu) / \sigma>0\}$ where $z$ represents maximum precipitation, $-\infty<\mu<\infty$ is the location parameter, $\sigma>0$ is the scale parameter and $-\infty<$ $\xi<\infty$ is the shape parameter. The latter describes the heaviness of the tail of the distribution: the larger the value of $\xi$, the heavier the tail of the distribution, the larger the probability of occurrence of extreme values. The subset of the GEV family distributions with $\xi=0$ is interpreted as the limit of the GEV as $\xi \rightarrow 0$, leading to the Gumbel distribution; the case with $\xi>0(\xi<0)$ correspond to the Fréchet (Weibull) family distributions. The parameters of the GEV are estimated in this study using maximum likelihood estimation 
(e.g., Coles, 2001) by means of the gevfit Matlab function, which also provides the corresponding $95 \%$ confidence intervals.

The quantiles of the GEV can be written as a function of the return period by inverting Eq. (2):

$z_{\mathrm{p}}=\left\{\begin{array}{ll}\mu-\frac{\sigma}{\xi}\left[1-\{-\ln (1-p)\}^{-\xi}\right] & \text { for } \xi \neq 0 \\ \mu-\sigma \ln \{-\ln (1-p)\} & \text { for } \xi=0\end{array}\right.$,

where $p$ is the probability that the maximum rainfall exceeds $z_{\mathrm{p}}$ and the return period $T$ is thus

$T=1 / p$.

Here, $z_{\mathrm{p}}$ is called the return level associated with the return period $T$, and it represents the rainfall amount which is expected to be exceeded on average once every $T$ blocks. The curve which shows the behavior of $z_{\mathrm{p}}$ against $T$ is called return level plot, and by $\log -\log$ transforming the $x$ axis, it is possible to focus on the tail behavior of the distribution. Even though in this study return level plots were obtained for each rainfall accumulation, warning region and regional statistic, here we only show maps of the return levels corresponding to given return periods, rainfall accumulation and regional statistics for each warning region. Since we need to get rainfall amounts corresponding also to sub-annual return periods, the monthly maxima of each regional statistic and warning region have been extracted from the 10-year data set. In fact, NowPAL must be able to issue alerts also for heavy rainfall episodes which are relatively frequent and not necessarily extreme. Our assumption is that the GEV parameters are constant within each warning region, and are motivated by the need to derive rainfall thresholds corresponding to specific return periods for the regions. The local maxima used for the statistical analysis have not necessarily been measured at the same place, but they might have occurred at different locations within the region of interest. As soon as they belong to the same warning region, they constitute the empirical observations for that region, and their actual position within the region is not taken into account. With this regional approach, the limited length of the monthly block is compensated by the large number of pixels within each region which might originate the regional maximum.

In order to guarantee the temporal independence of maxima of blocks as short as months, a minimum lag time of $48 \mathrm{~h}$ among maxima occurring in 2 successive months but close in time was imposed for accumulations ranging from $1 \mathrm{~h}$ to 1 day. In fact, Fukutome et al. (2015) and Barton et al. (2016) found that this is the maximum declustering run length in Switzerland for hourly and daily precipitation, respectively. For 2-day rainfall accumulations, such lag time was extended to $72 \mathrm{~h}$. In case of maxima of 2 separate months occurring within this lag time, the largest was assigned to the corresponding month, while the smallest was substituted with the second maximum of the other month. Moreover, the analysis was performed separately for the warm (May-October) and cold (November-April) seasons in order to reduce the effect of seasonality on the choice of maxima, assuming that the GEV parameters are constant within each season. Thus, a total of 6 (months) $\times 10$ (years) $=60$ monthly maxima are considered for each season. Since the estimation of solid precipitation from radar suffers from underestimation, the cold season return levels might result underestimated as well. However, we should mention that the NowPAL system was designed to issue alerts mainly in summer, when liquid precipitation makes rainfall estimates more reliable and precipitation intensities are indeed more critical.

\section{Regional extreme rainfall analysis}

In this section the main results of the regional extreme rainfall analysis are reported.

\subsection{Return level plots}

As introduced in Sect. 2.3, the GEV distribution has been fitted to seasonal monthly maxima of rainfall for each warning region, regional statistic and for several temporal accumulations. As an example, Fig. 4 shows the GEV fitted to warm season maxima of $1 \mathrm{~h}$ mean rainfall measured in the Schaffhausen warning region $\left(273 \mathrm{~km}^{2}\right)$, and the return level curve with empirical observations for the maximum of regional rainfall computed after a spatial aggregation of the original field with a moving window of $3 \times 3,5 \times 5$ and $7 \times 7 \mathrm{~km}^{2}$. As expected, return levels decrease with increasing the size of the moving window used to smooth the original rainfall field, with the regional mean providing the smallest return levels. For example, a storm which produces about $20 \mathrm{~mm}$ of rainfall on average in the region has the same 3year return period as a storm which causes about $70 \mathrm{~mm}$ of rainfall in a $9 \mathrm{~km}^{2}$ area within the region. Another feature of the regional return level plot, which is common also to other Swiss regions, is that the confidence of the GEV fitting increases with increasing the averaging area, with the mean rainfall providing the smallest confidence interval.

\subsection{Return level maps}

Return level plots for a given rainfall accumulation, like that shown in Fig. 4, provide a fast tool to associate a rainfall amount with each warning region for a particular return period and regional statistic. Maps showing for each warning region the return level corresponding to 3-, 6- and 12-month (or 0.5-, 1- and 2-season) return periods for 3, 6, 12 and $24 \mathrm{~h}$ rainfall for the warm season are presented in Fig. 5. It should be noted that the maps of the first line of Fig. 5 are not quantitatively comparable with the other maps, since they were derived from radar and not from CombiPrecip as the other maps of the figure (see Sect. 2.1). The regional statistic used to obtain the maps is $\max \left(R 5\left(\operatorname{tot}_{i}\right)_{j}\right)$, i.e., the maximum of 

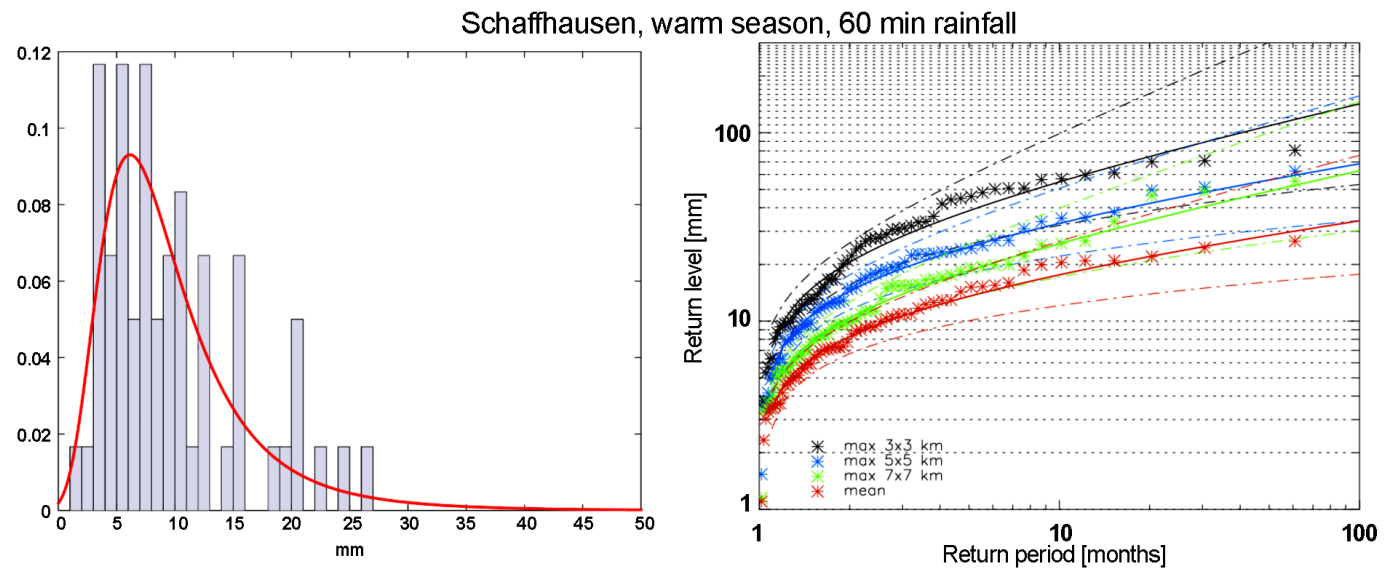

Figure 4. Histogram of warm season monthly maxima of mean rainfall in the Schaffhausen region $\left(273 \mathrm{~km}^{2}\right)$ with GEV fit (left), and corresponding return level plot for several regional statistics (right).
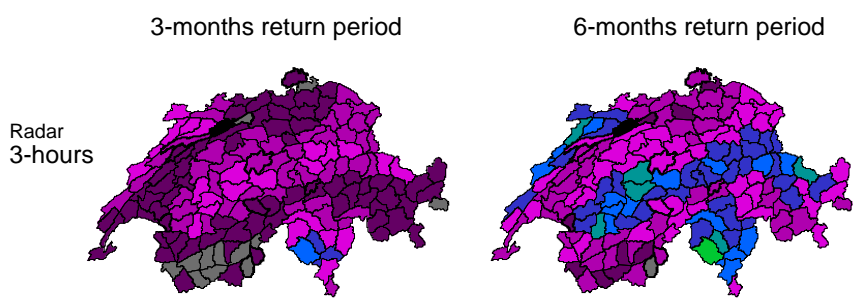

12-months return period
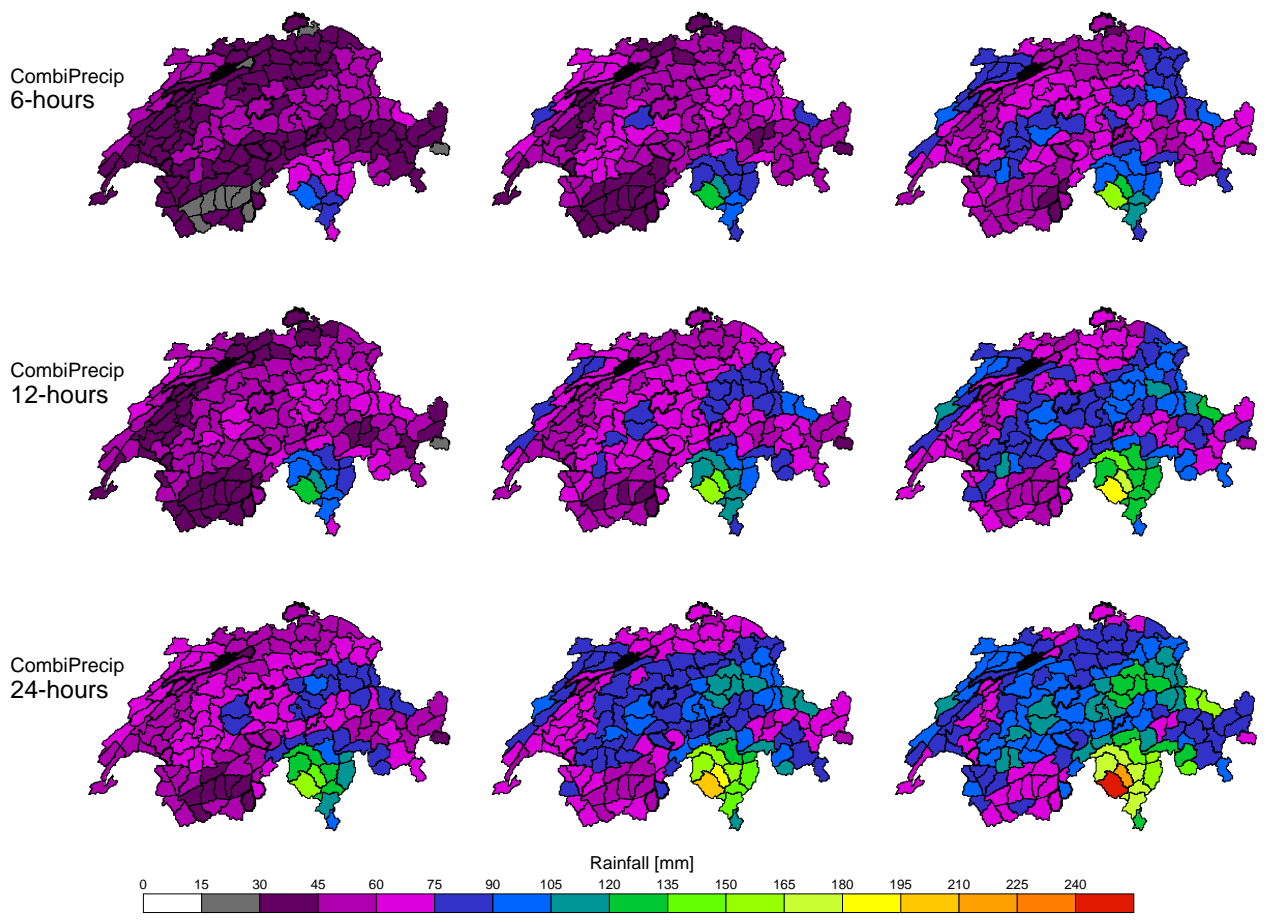

Figure 5. Maps of warm season return levels for the Swiss warning regions corresponding to 3-, 6- and 12-month return periods, for 3, 6, 12 and $24 \mathrm{~h}$ rainfall accumulations. 

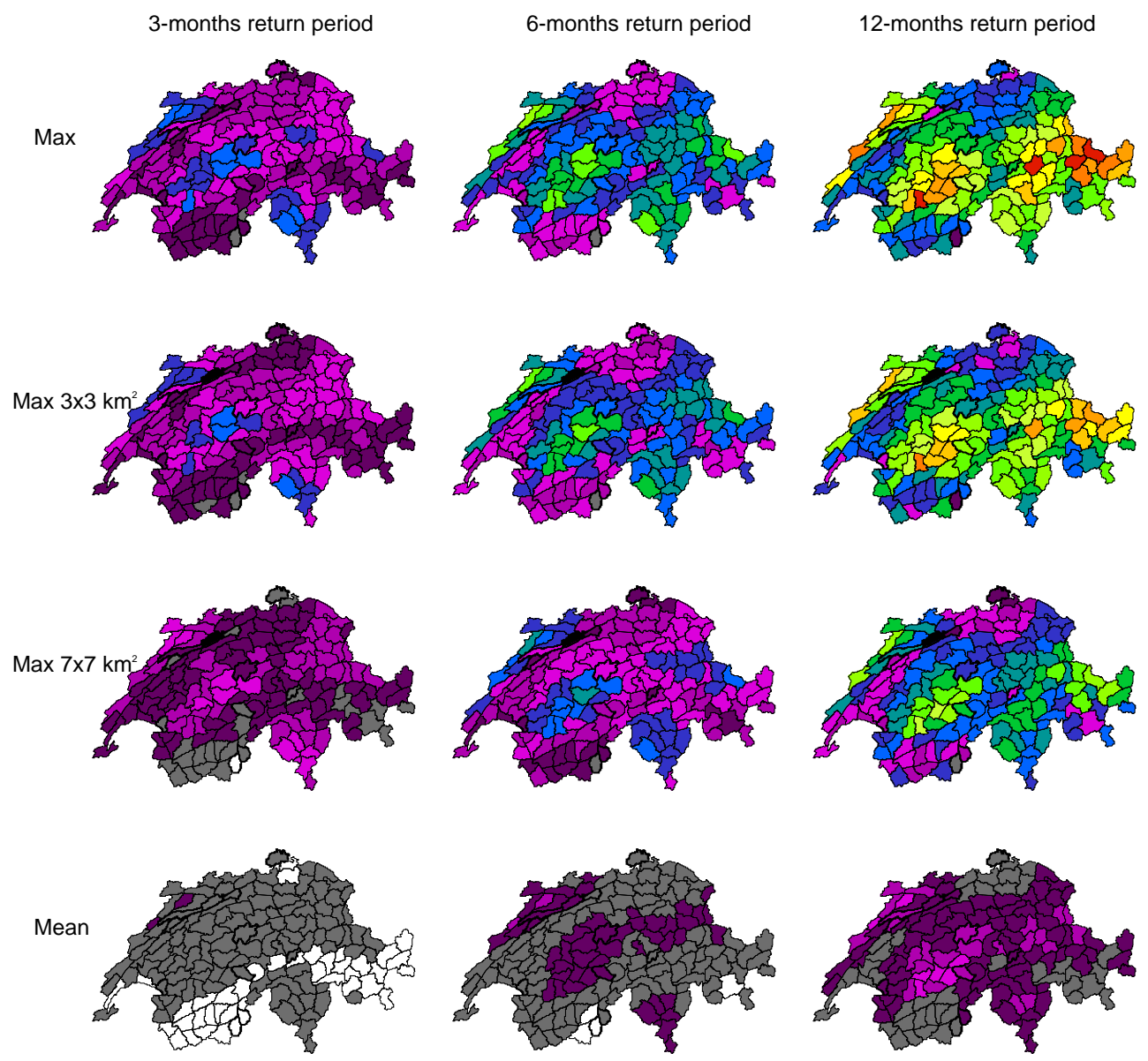

Rainfall [mm]

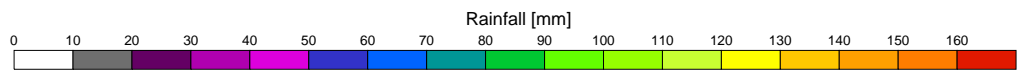

Figure 6. Maps of warm season $1 \mathrm{~h}$ return levels for the Swiss warning regions corresponding to 3-, 6- and 12-month return periods, for several regional statistics.

regional rainfall over an area of $25 \mathrm{~km}^{2}$; thus, the original rainfall field was spatially aggregated with a moving window of $5 \times 5 \mathrm{~km}^{2}$ before extracting the monthly maximum within each region. The borders of the regions for which more than half of the pixels were identified as affected by residual ground clutter are indicated with a thicker contour in the map, indicating that the corresponding return levels were derived only from a small part of the region. Only for one region (shaded in black in the figure) in the Jura Mountains was it not possible to derive return levels, as more than $95 \%$ of it was affected by residual ground clutter. Since monthly maxima are considered for each 6-month season, the rainfall amount corresponding to a return period of 3 months is expected to occur on average twice a summer (May-October), while the rainfall with a 6-month return period occurs on average every summer; similarly, a 12-month return period is associated with a rainfall amount expected on average every two summers. Figure 5 shows that the largest return levels occur south of the Alps in the Ticino region for $6 \mathrm{~h}$ or longer precipitation accumulations, whereas for $3 \mathrm{~h}$ rainfall the largest values are observed north of the Alps, in particular in the western Bernese Alps as well as in the Jura Mountains and northeast Switzerland. The central alpine chain exhibits return levels lower than the southerly and northerly alpine slopes, in particular Cantons of Valais and Grisons show very low return levels for accumulations longer then $3 \mathrm{~h}$. It could be argued that the maxima of rainfall of these two latter regions are underestimated because the 3GEN of radars had low visibility there; however, this is not the case since CombiPrecip data are used in the analysis. The Jura Mountains, on the other hand, show larger values than the Swiss Plateau, with rainfall totals comparable with those observed over the northern Alps. The effect of varying the regional statistic on the return levels is shown in Fig. 6 for $1 \mathrm{~h}$ radar rainfall accumulation for return periods of 3, 6 and 12 months. The regional statistics employed for the analysis are $\max \left(R 1\left(\operatorname{tot}_{i}\right)_{j}\right), \max \left(R 3\left(\operatorname{tot}_{i}\right)_{j}\right), \max \left(R 7\left(\operatorname{tot}_{i}\right)_{j}\right)$ and $\bar{R}\left(\operatorname{tot}_{i}\right)_{j}$. As it was also observed in Fig. 4, return levels in- 


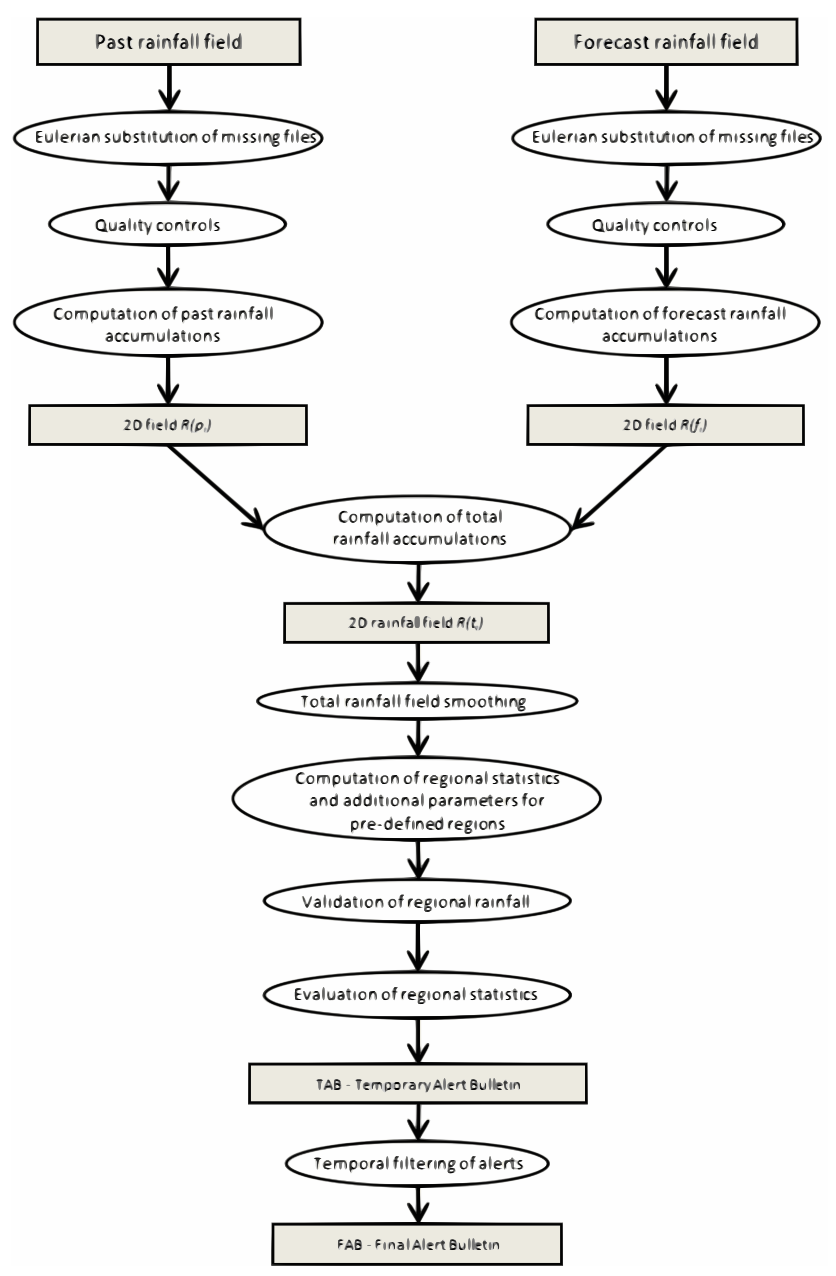

Figure 7. Flowchart of NowPAL algorithm.

crease with decreasing the averaging area, with the mean rain providing the lowest values. The spatial distribution of the return levels for $1 \mathrm{~h}$ rainfall is generally similar to those of $3 \mathrm{~h}$ precipitation, with some regions of Engadin in the Canton of Grisons showing values as large as the western Bernese Alps. The spatial distribution of the hourly and daily return levels shown in Fig. 5 and 6 mirrors the findings of previous alpine climatologies (e.g., Frei and Schär, 1998; Isotta et al., 2014; Fukutome et al., 2015). Figure 6, moreover, shows that the regional statistic considered for the extreme value analysis has a huge impact on the thresholds which should be used by NowPAL to issue alerts, and therefore it should be carefully chosen when designing a regional alert system, depending on the nowcasting application and specific customer needs.

\section{NowPAL algorithm description}

This section describes in detail the real-time NowPAL algorithm, whose structure is schematically illustrated in Fig. 7.
NowPAL is designed to monitor rainfall accumulations within the regions of interest and to automatically issue the alerts if the thresholds are exceeded depending on the requirements of the customers. Using $N$ to indicate the rainfall accumulation periods in which the customers are interested, $p_{i}=p_{1}, p_{2}, . ., p_{N}$ to indicate the minutes of the past and $f_{i}=f_{1}, f_{2}, . ., f_{N}$ to indicate the minutes of the future of the rainfall accumulations, tot ${ }_{i}=p_{i}+f_{i}$ represent the number of minutes for which total rainfall accumulations are computed. If $M$ is the number of predefined geographical regions associated to each customer, $\operatorname{reg}_{j}=\operatorname{reg}_{1}, \operatorname{reg}_{2}, . ., \operatorname{reg}_{M}$ are the regions which will be monitored for a specific client; thus, an alert $\mathrm{al}_{i j}$ corresponds to each temporal period of tot ${ }_{i}$ minutes for the region reg ${ }_{j}$, in case of threshold exceedance. The system runs every $\tau_{i}$ minutes, by taking the most updated past and forecast rainfall fields for total rainfall computation. 


\subsection{Computation of rainfall totals}

This subsection briefly describes the part of the NowPAL algorithm responsible for computing past, forecast and total rainfall accumulation fields.

In order to derive past rainfall accumulations, NowPAL makes use of the radar QPE or CombiPrecip rainfall maps of the last $p_{N}$ minutes (see Sect. 2.1). Before computing summations, the algorithm checks if 5 min radar images are missing within the longest accumulation period. The gaps due to missing images are filled by replicating the last available rainfall field, assuming that within a short period the rainfall field does not significantly change (Eulerian persistence approach, Germann and Zawadzki, 2004). In case of a gap caused by consecutive missing images, the second missing image is assumed to be the average of the last two available radar images, excluding those replicated with Eulerian persistence. Similarly, the third missing rainfall field is created by averaging the three last images not already replicated in this process. This action is performed for a configurable number of times (e.g., three in the current version of the system). Once the data gaps have been filled, some plausibility controls are performed, with the aim to check whether the rainfall values measured every $5 \mathrm{~min}$ by the radar or estimated by CombiPrecip are within a range of plausible values. The average rainfall and the fraction of area with rainfall larger than a configurable threshold within the Swiss radars domain and within circles around the radars have to be lower than configurable thresholds, as specified in a configuration file. The images which do not show plausible rainfall values, which might be due to radar artifacts or other errors, are discarded and substituted with the previous ones, following the Eulerian persistence approach described above. Then the temporal consistency between successive radar or CombiPrecip images is checked: some statistical indices between the last radar image and the previous one, and between the previous one and the one before are computed (correlation, bias, variation of fraction of area with rainfall larger than a configurable threshold). These statistical indices are computed taking into account the rainfall measured only within Switzerland. Then, the difference between the indices relative to the two pairs of images is computed and evaluated: if it is larger than configurable thresholds, the last radar image is discarded and substituted with the Eulerian persistence approach. It is worth highlighting that Eulerian replacement of missing and implausible images occurs very rarely, as more than $99.9 \%$ of 5 min radar images of a year are regularly produced in real time, and they contain plausible rainfall values. The past rainfall accumulations $R\left(p_{i}\right)$ are then computed at the pixel level, by also taking into account the radar images replicated or substituted with Eulerian persistence for each accumulation period. Four quality flags, accounting for files' availability and the outcome of the Eulerian replacement of missing and low-quality files, are associated with the past rainfall accumulation image and reported in the final alert bulletin.

Section 2.2 already introduced the nowcasting tools which provide forecast rainfall fields for NowPAL. In this step of the algorithm, the most recent available forecasts for the next $f_{N}$ minutes are taken into account to compute the temporal aggregation. Similarly to what is done for past rainfall accumulations, the forecast rainfall data are also checked for possible missing images and quality controlled, and the same approach of Eulerian persistence substitution developed for past rainfall accumulations is implemented also for forecasts. Then, forecast rainfall accumulations $R\left(f_{i}\right)$ are computed as a simple summation at the pixel level, and five quality flags, giving information about files availability, quality controls outcome and the delay of the forecast with respect to current time, are assigned to these fields and reported in the final alert bulletin.

Finally, total rainfall fields $R\left(\right.$ tot $\left._{i}\right)$ are obtained by summing the most updated past and forecast rainfall accumulations at the pixel level.

\subsection{Issuance of regional alerts}

This subsection briefly describes the part of the algorithm responsible for issuing alerts for predefined geographical regions. A number of consecutive actions is done by the system:

1. Total rainfall field smoothing. In this step, the algorithm performs a spatial aggregation of the total precipitation field with a square moving window of configurable size, producing $R S\left(\operatorname{tot}_{i}\right)$, a smoothed total rainfall field which is used by the system if the regional statistic chosen to evaluate regional rainfall is $\max \left(R S\left(\operatorname{tot}_{i}\right)_{j}\right)$ (see Sect. 2.3). In the current configuration of NowPAL, aimed to issue alerts for the Swiss warning regions, the area of the square moving window is set to $5 \times 5$ and $7 \times 7 \mathrm{~km}^{2}$, with the largest window being used for the longest accumulations.

2. Computation of regional statistics. In this step, the regional statistics $\operatorname{stat}\left(R\left(\operatorname{tot}_{i}\right)_{j}\right)$ introduced in Sect. 2.3, representative of the total rainfall distribution $R\left(\operatorname{tot}_{i}\right)_{j}$ over each region $j$, are computed. If the required statistic is $\max \left(R S\left(\operatorname{tot}_{i}\right)_{j}\right)$, the maximum of $R S\left(\operatorname{tot}_{i}\right)$ within each region is derived. Moreover, some additional statistics are also computed, in order to provide further information about the regional rainfall distribution; these include the percent of $R S\left(\operatorname{tot}_{i}\right)_{j}$ caused by $R S\left(p_{i}\right)_{j}$, the fraction and the area of each region with rainfall larger than a configurable threshold, the average regional rainfall, the maximum and the total of regional rainfall.

3. Validation of regional rainfall. In this step of the algorithm, $R S\left(\text { tot }_{i}\right)_{j}$ is quality controlled through a plausibility test: for each region, a maximum value of mean 
hourly rainfall accumulation is allowed. Moreover, a coefficient from 0 to 1 should also be defined, as this number is multiplied by the maximum mean hourly rainfall and by the number of hours of tot ${ }_{i}$, to obtain the maximum possible mean regional rainfall for accumulations longer than hourly. This regional plausibility control also results in a quality flag which is reported in the final alert bulletin.

4. Evaluation of regional statistics. The regional statistics $\operatorname{stat}\left(R\left(\operatorname{tot}_{i}\right)_{j}\right)$ are then compared with the threshold values in order to assess if alerts need to be issued in case of threshold exceedance. This step results in a temporary alert bulletin $\mathrm{TAB}_{i}$, which is a list of the regions with the relative alert levels, regional and additional statistics. $\mathrm{TAB}_{i}$ is produced for each $R\left(\operatorname{tot}_{i}\right)$ total rainfall accumulation, and it is updated every $\tau_{i}$ minutes.

5. Temporal filtering of alerts. Since during a heavy precipitation event the thresholds corresponding to the alerts might be continuously exceeded for a particular region for a long period, the aim of temporal filtering is to identify the alerts reported in $\mathrm{TAB}_{i}$ which should receive special attention by the customer because they can be considered as new. For this scope, a latency time lat $_{i}$ has to be defined for each total rainfall accumulation tot ${ }_{i}$, representing the temporal period during which NowPAL checks if an alert has been previously issued for the same region; if this is the case, the system considers the latest alert as new only if its level is larger from that of the previous alerts found in $\mathrm{TAB}_{i}$, or if the latency time since the last new alert has passed. Then, a special flag is assigned to alerts recognized as new, and reported in a final alert bulletin $\mathrm{FAB}_{i}$ for each alert. By disseminating only the alerts identified as new in $\mathrm{FAB}_{i}$, NowPAL can thus control how often the customers should be alerted in case of continuous rainfall threshold exceedance.

Figure 8 provides an example of visualization of the alerts issued by NowPAL for the 159 Swiss warning regions. Different colors denote the level of the alerts, whereas the regions with a thick border are those for which a new alert is identified at the current time.

\section{Urban case study}

This section presents a case study for the urban flood which occurred in the city of Schaffhausen in Switzerland on 2 May 2013. The aim of this short analysis is to provide a concrete example to make the reader familiar with the regional alerting tool presented in this paper.

On 2 May 2013, a moist southwesterly, large-scale circulation associated with an upper-level trough favored the development of multicell thunderstorms in Switzer-

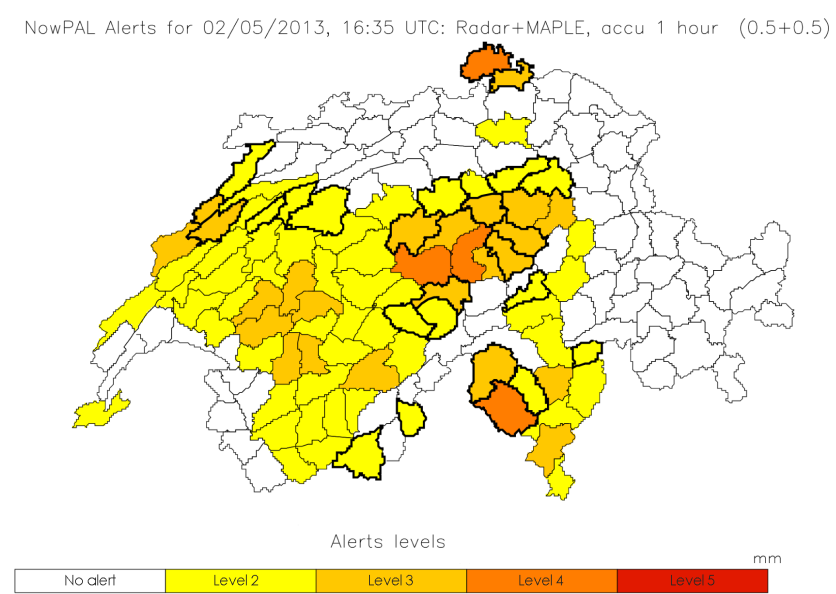

Figure 8. Example of NowPAL output: map of Swiss warning regions with corresponding alert levels for $1 \mathrm{~h}$ accumulation.

land, with damages being reported in many Swiss cantons. As shown in Fig. 9, between 16:00 and 16:30 UTC, a mesoscale convective system rapidly developed over the city of Schaffhausen, which was heavily affected by intense precipitation. Widespread flooding through the city and the surrounding areas was reported, resulting in CHF 25 million worth of damages. From 16:20 to 17:20 UTC, the local rain gauge measured a total rainfall of $51.4 \mathrm{~mm}$, with $46.6 \mathrm{~mm}$ falling in $30 \mathrm{~min}$ and a peak intensity of $32.8 \mathrm{~mm}$ within 10 min (16:40-16:50 UTC); small hail has also been observed. NowPAL was run for the area of Schaffhausen municipality ( $44 \mathrm{~km}^{2}$, see Fig. 9 ), by combining the radar and MAPLE data which would have been available in real time; both fields are produced within $3 \mathrm{~min}$ after the nominal time. The accumulation period is $1 \mathrm{~h}$ ( $30 \mathrm{~min}$ past $+30 \mathrm{~min}$ future), and the rainfall thresholds are 20,30, 40 and $50 \mathrm{~mm}$, corresponding to warm-season return periods of 6 months, 1,2 and 4 years for $\max \left(R 7\left(\operatorname{tot}_{i}\right)_{j}\right)$ within the larger Schaffhausen warning region. The regional statistic accounting for the total rainfall measured within the Schaffhausen municipality was set to the mean, updating frequency was $5 \mathrm{~min}$ and latency time $10 \mathrm{~min}$. Figure 10 shows the behavior over time of radar $5 \mathrm{~min}$ rain rate, $30 \mathrm{~min}$ past (radar) and forecast (MAPLE) accumulation, and $1 \mathrm{~h}$ total rainfall for Schaffhausen. TAB and FAB alert levels are also reported, with the new alerts of FAB highlighted in bold. NowPAL issued an alert level 1 at 16:10, when total rainfall exceeded the lower threshold of $20 \mathrm{~mm}$. Alert level increased to 3 at 16:15, and to 4 at 16:20. For the largest part of the storm, MAPLE predictions were contributing most to total precipitation accumulation, and they were indeed overestimating rainfall quantity. The maximum mean rainfall intensity was observed between 16:45 and 17:00. Then rainfall intensity started to slowly decrease, as well as MAPLE forecasts. At 17:10, alert level dropped to 3 . After $17: 15$, past radar rainfall accumulation was larger than MAPLE forecasts. The effect of temporal 


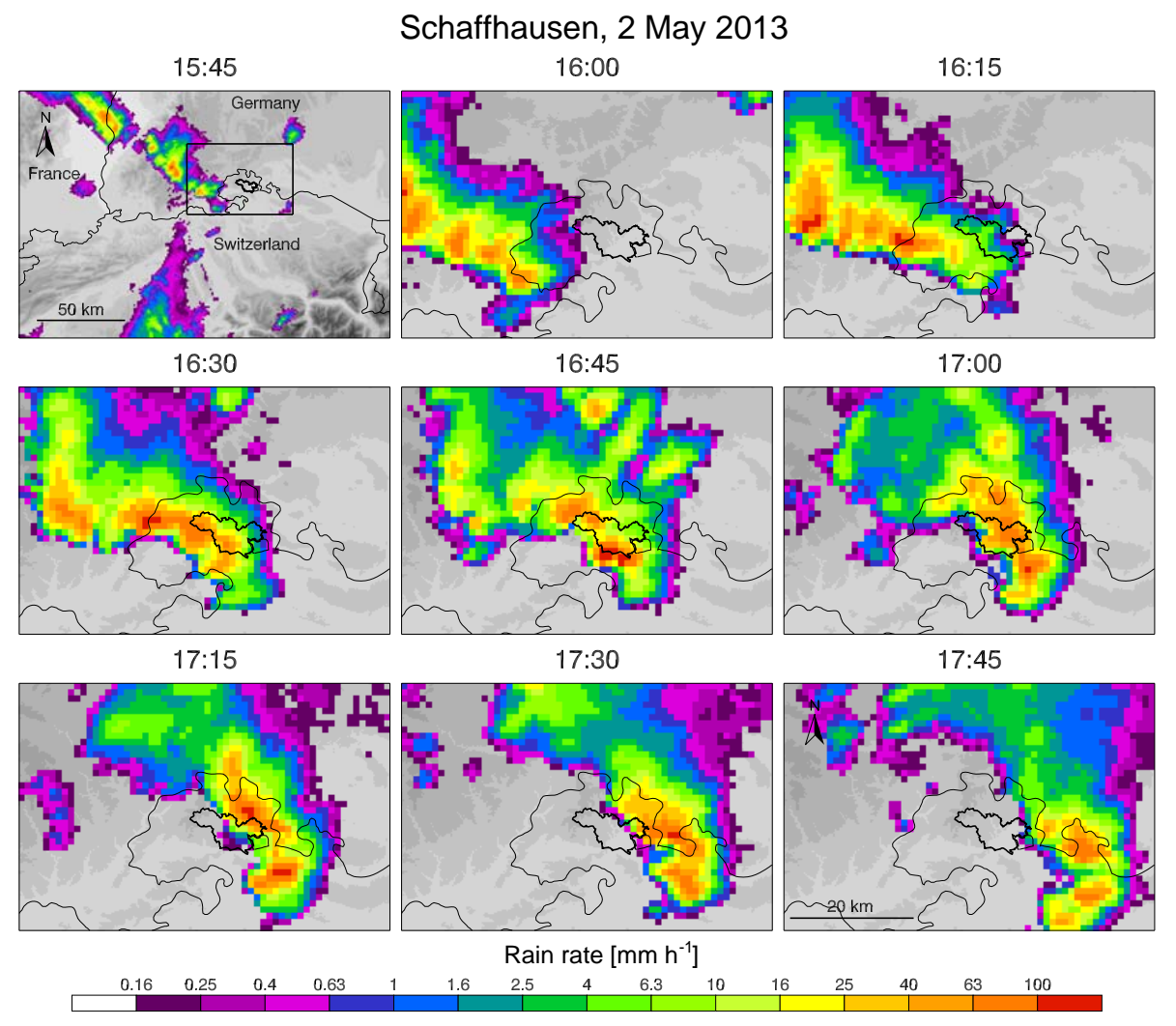

Figure 9. Time series of radar images showing the storm that hit the city of Schaffhausen on 2 May 2013. The first panel shows the largescale rainfall pattern, with the box indicating the area of the other panels, a smaller region centered on Schaffhausen municipality $\left(44 \mathrm{~km}^{2}\right)$, whose boundaries are also shown in bold.

filtering of alerts can also be seen in Fig. 10. For example, even though at 17:15 the last radar observations and MAPLE forecasts were corresponding to alert level 2, alert level 3 was reported in FAB because the latency time was not yet expired (see Sect. 4.2). During this storm, end users would have received the alert levels marked in bold in the figure, in order to deal with a manageable amount of information.

Nowcasting of thunderstorms is done at MeteoSwiss by TRT, an automatic algorithm for the detection, tracking, characterization and extrapolation of convective cells (Hering et al., 2008). The system includes a thunderstorm severity ranking product, which, based on cell attributes such as vertically integrated liquid water, mean of $45 \mathrm{dBZ}$ echo top altitude, maximum reflectivity and cell size above $55 \mathrm{dBZ}$, classifies each cell in the four categories of very weak, weak, moderate and severe. If a thunderstorm is classified as moderate for two consecutive $5 \mathrm{~min}$ radar scans, a warning is issued for the regions affected by the cell in the following hour according to radar extrapolation. Unfortunately, MeteoSwiss did not issue any thunderstorm warning on 2 May 2013 for Schaffhausen, as the storm did not reach the category moderate. This means that the convective cell was not as vertically developed as severe thunderstorms usually are, although it produced a large quantity of rainfall and small hail parti- cles. The Schaffhausen storm is typical of those that are not correctly classified as severe by common thunderstorm radar tracking systems, because of their limited vertical depth and convective features. For these storms, which constitute a real challenge for forecasters, nowcasting systems based on extrapolation of radar images, such as INCA or MAPLE, are the most valid solution. The case study presented here shows that NowPAL is needed to combine these forecasts with information about recent past accumulation, possibly increasing the lead time of warnings.

\section{Conclusions}

This paper presented a regional extreme rainfall analysis based on 10 years of radar data for the 159 Swiss warning regions and introduced NowPAL, a tool for issuing regional heavy precipitation alerts. The statistical analysis and the nowcasting system are strictly related, since the first aimed to provide the rainfall thresholds needed by the second to issue the alerts. Thus, the paper offers an intuitive and practical solution for both implementing a regional rainfall alert system and for choosing the rainfall thresholds corresponding to the alert levels. 


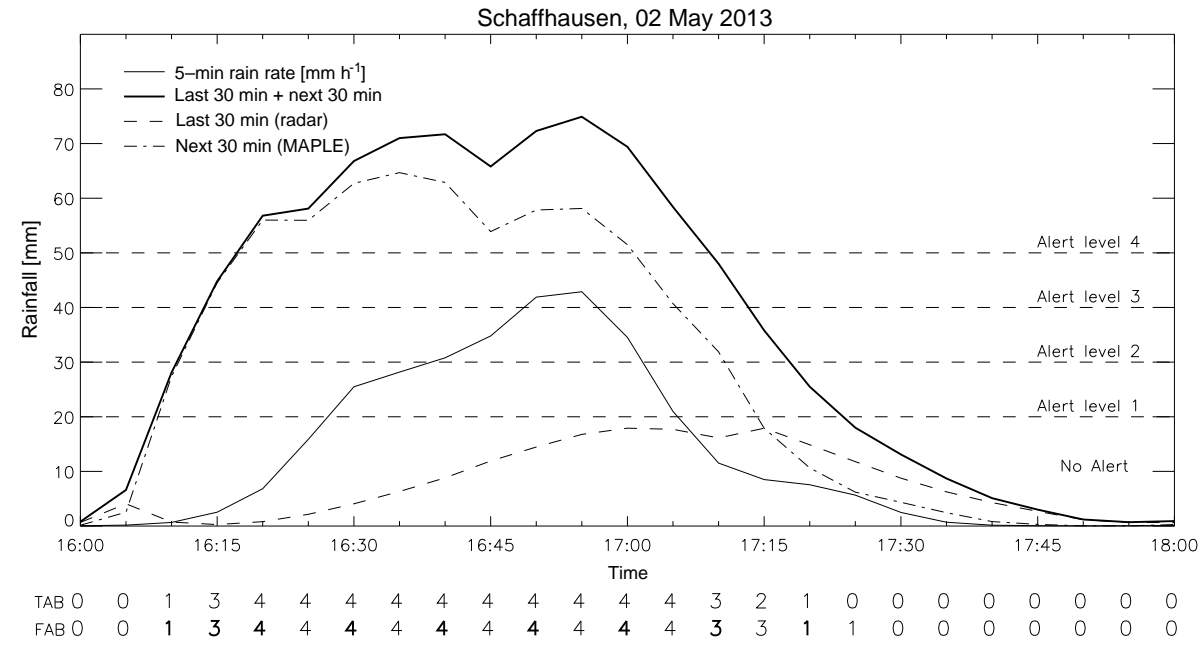

Figure 10. Behavior over time of the $5 \mathrm{~min}$ rain rate, $30 \mathrm{~min}$ past (radar) and forecast (MAPLE) accumulation, and $1 \mathrm{~h}$ total rainfall averaged over the Schaffhausen municipality. NowPAL TAB and FAB alert levels are also indicated, with the new alerts of FAB highlighted in bold. See text for details.

Warm and cold seasons' monthly maxima of several statistical quantities describing the rainfall within the regions were fitted to a generalized extreme value distribution for 1 , $3,6,12$ and $24 \mathrm{~h}$ rainfall aggregations. Return level plots, showing the precipitation amounts corresponding to monthly return periods, were obtained for each accumulation period, warning region and for several regional statistics, i.e., the statistical quantities used to describe the regional rainfall distribution. Thus, the high spatial resolution of radar precipitation fields is fully exploited in this analysis. A large variability of return levels was found between the Swiss warning regions, and it was shown that their spatial distribution depends on the duration of the aggregation period, independent on the regional statistics. For accumulations of $3 \mathrm{~h}$ or shorter, the largest return levels are found in the western Bernese Alps and in the Alps of Cantons of Fribourg and Vaud, whereas for longer durations, Ticino exhibits the largest values. The inner alpine chain shows the lowest values, in agreement with previous climatologies.

Return levels are used as rainfall thresholds and associated to different alert levels by NowPAL. In fact, our assumption is that an alert of a given level should be expected the same number of times during a season in every warning region. Under this assumption, the extreme rainfall analysis objectively provides the threshold values for each warning region and for a given regional statistic, once the return periods corresponding to the alert levels have been fixed. The alerts of NowPAL can be based on the precipitation observed or forecast over the region, or on the sum of measured and predicted regional rainfall. The latter quantity has a fundamental importance for nowcasting applications for which antecedent rainfall is as important as predicted precipitation, such as urban or small river floods predictions. The dependance of return levels on regional statistic indicates that the latter is a key element of a regional alert system, which should be carefully chosen. In order to answer the needs of end users which might be interested in monitoring different aspects of precipitation, in fact, the system should be able to monitor rainfall in real time over several spatial and temporal scales. The algorithm, which was described in detail in the paper, also includes a practical method to control the number of alerts issued in case of continuous threshold exceedance during a prolonged precipitation event. For all these reasons, NowPAL was designed as a fully configurable system, which can be adapted to the different needs of the end users.

NowPAL finds a natural application in the prediction of urban flooding. In order to make the reader familiar with the system for this kind of application, the urban flood which occurred in the city of Schaffhausen in 2013 was analyzed in detail. Even though a verification of NowPAL alerts is not within the scope of the paper, since its performance strongly depends on the quality of the ingested QPE and the skill of the adopted forecasting system, the analysis shows that the tool offers a practical method to efficiently combine past and forecast rainfall fields in real time and to continuously monitor total rainfall accumulation over a city. Moreover, the case study suggests that the system might have complementary skill with respect to radar-based thunderstorm nowcasting systems for storms which do not show a clear convective signature because of their limited vertical development. For such storms, combining two-dimensional measured and forecast precipitation fields can add lead time to the heavy rainfall warnings.

A comparison of extreme rainfall analyses derived from radar and rain gauges deserves future investigations. Even though rain gauges typically provide longer and more homogeneous data records with respect to radar, they suffer from limited spatial representativeness. Radar data with $1 \mathrm{~km}^{2}$ 
spatial resolution, on the other hand, offer the opportunity to investigate in detail not only the geographical variability of extreme precipitation but also the behavior of extremes over different spatial scales. Thus, extreme rainfall statistics derived from radar and rain gauges provide complementary information, and the specific application should drive the choice about the source of data to take into consideration. The NowPAL system presented here is an excellent example of a nowcasting application which requires a radar-based statistical analysis, for which rain gauges would have provided only very limited information.

\section{Data availability}

MeteoSwiss Radar and CombiPrecip maps are freely available for research purposes upon request at meteosvizzera@meteosvizzera.ch.

Acknowledgements. We would like to thank N. Besic, M. Boscacci, P. Ambrosetti, F. Marra, A. Schindler and S. Fukutome for their helpful comments. Thanks are due also to I. Sideris for providing MAPLE data for the urban case study.

Edited by: C. Onof

\section{References}

Alfieri, L., Velasco, D., and Thielen, J.: Flash flood detection through a multi-stage probabilistic warning system for heavy precipitation events, Adv. Geosci., 29, 69-75, doi:10.5194/adgeo-29-69-2011, 2011.

Alfieri, L., Salamon, P., Pappenberger, F., Wetterhall, F., and Thielen, J.: Operational early warning systems for water-related hazards in Europe, Environ. Sci. Policy, 21, 35-49, 2012.

Allen, R. J. and DeGaetano, A. T.: Considerations for the use of radar-derived precipitation-estimates in determining return intervals for extreme areal precipitation amounts., J. Hydrol., 315, 203-219, 2005.

Atencia, A., Rigo, T., Sairouni, A., Moré, J., Bech, J., Vilaclara, E., Cunillera, J., Llasat, M. C., and Garrote, L.: Improving QPF by blending techniques at the Meteorological Service of Catalonia, Nat. Hazards Earth Syst. Sci., 10, 1443-1455, doi:10.5194/nhess-10-1443-2010, 2010.

Barton, Y., Giannakaki, P., von Waldow, H., Chevalier, C., Pfahl, S., and Martius, O.: Clustering of regional-scale extreme precipitation events in Southern Switzerland., Mon. Weather Rev., 144, 347-369, 2016

Bowler, N. E., Pierce, C. E., and Seed, A. W.: STEPS: A probabilistic precipitation forecasting scheme which merges an extrapolation nowcast with downscaled NWP, Q. J. Roy. Meteorol. Soc., 132, 2127-2155, 2006.

Coles, S.: An Introduction to Statistical Modeling of Extreme Values, Springer-Verlag London, 2001.

Collier, C. G.: Flash flood forecasting: What are the limits of predictability?, Q. J. Roy. Meteorol. Soc., 133, 3-23, 2007.
Durrans, S. R., Julian, L. T., and Yekta, M.: Estimation of deptharea relationships using radar-rainfall data, J. Hydrol. Eng., 7, 356-367, 2002.

Fouchier, C., Mériaux, P., Atger, F., Ecrepont, S., Liébault, F., Bertrand, M., Bel, C., Batista, D., Azemard, P., Saint-Martin, C., and Javelle, P.: Implementation of a real-time warning and mapping system for natural hazards triggered by rainfall in mountainous and Mediterranean areas of Southeastern France, in: Proc. 10th Intern. Workshop on Precipitation in Urban Areas, ETH Zürich, Institute of Environmental Engineering, 2015.

Frei, C. and Schär, C.: A precipitation climatology of the Alps from high-resolution rain-gauge observations, Int. J. Climatol., 18, 873-900, 1998.

Fukutome, S., Liniger, M. A., and Süveges, M.: Automatic threshold and run parameter selection: a climatology for extreme hourly precipitation in Switzerland., Theor. Appl. Climatol., 120, 403-416, 2015.

Germann, U. and Joss, J.: Variograms of Radar Reflectivity to Describe the Spatial Continuity of Alpine Precipitation, J. Appl. Meteorol., 40, 1042-1059, 2001.

Germann, U. and Joss, J.: Mesobeta Profiles to Extrapolate Radar Precipitation Measurements above the Alps to the Ground Level, J. Appl. Meteorol., 41, 542-557, 2002.

Germann, U. and Zawadzki, I.: Scale-dependence of the Predictability of Precipitation From Continental Radar Images. Part I: Description of the Methodology, Mon. Weather Rev., 130, 2859-2873, 2002.

Germann, U. and Zawadzki, I.: Scale-dependence of the Predictability of Precipitation From Continental Radar Images. Part II: Probability forecasts, J. Appl. Meteorol., 43, 74-89, 2004.

Germann, U., Galli, G., Boscacci, M., and Bolliger, M.: Radar precipitation measurement in a mountainous region, Q. J. Roy. Meteorol. Soc., 132, 1669-1692, 2006.

Germann, U., Boscacci, M., Gabella, M., and Sartori, M.: Radar design for prediction in the Swiss Alps, Meteorological Technology International, 4, 42-45, 2015.

Giannecchini, R., Galanti, Y., and D’Amato Avanzi, G.: Critical rainfall thresholds for triggering shallow landslides in the Serchio River Valley (Tuscany, Italy), Nat. Hazards Earth Syst. Sci., 12, 829-842, doi:10.5194/nhess-12-829-2012, 2012.

Golding, B.: Nimrod: A system for generating automated very short range forecasts, Meteorol. Appl., 5, 1-16, 1998.

Guzzetti, F., Peruccacci, S., Rossi, M., and Stark, C. P.: Rainfall thresholds for the initiation of landslides in central and southern Europe, Meteorol. Atmos. Phys., 98, 239-267, 2007.

Haiden, T., Kann, A., Wittmann, C., Pistotnik, G., Bica, B., and Gruber, C.: The Integrated Nowcasting through Comprehensive Analysis (INCA) system and its validation over the Eastern Alpine region, Weather Forecast., 26, 166-183, 2011.

Hering, A. M., Germann, U., Boscacci, M., and Sénési, S.: Operational nowcasting of thunderstorms in the Alps during MAP DPHASE, in: The Fifth European Conference on Radar in Meteorology and Hydrology, Helsinki, Finland, 2008.

Isotta, F. A., Frei, C., Weilguni, V., Tadić, M. P., Lassegues, P., Rudolf, B., Pavan, V., Cacciamani, C., Antolini, G., Ratto, S. M., Munari, M., Micheletti, S., Bonati, V., Lussana, C., Ronchi, C., Panettieri, E., Marigo, G., and Vertačnik, G.: The climate of daily precipitation in the Alps: development and analysis of a high- 
resolution grid dataset from pan-Alpine rain-gauge data, Int. J. Climatol., 34, 1657-1675, 2014.

Javelle, P., Demargne, J., Defrance, D., Pansu, J., and Arnaud, P.: Evaluating flash-flood warnings at ungauged locations using post-event surveys: a case study with the AIGA warning system, Hydrolog. Sci. J., 59, 1390-1402, 2014.

Joss, J. and Lee, R.: The application of radar-gauge comparisons to operational precipitation profile corrections, J. Appl. Meteorol., 34, 2612-2630, 1995.

Knechtl, V.: Flash-flood early warning tool. Use of intensityduration-frequency curves for flash-flood warning in Southern Switzerland and forecast skill evaluation, MsC Thesis, ETH Zürich, 2013.

Kolmogorov, A.: Sulla determinazione empirica di una legge di distribuzione, G. Ist. Ital. Attuari, 4, 83-91, 1933.

Mandapaka, P. V., Germann, U., Panziera, L., and Hering, A.: Can lagrangian extrapolation of radar fields be used for precipitation nowcasting over complex alpine orography?, Weather Forecast., 27, 28-49, 2012.

Marra, F. and Morin, E.: Use of radar QPE for the derivation of Intensity-Duration-Frequency curves in a range of climatic regimes, J. Hydrol., 531, 427-440, 2015.

Martina, M. L. V., Todini, E., and Libralon, A.: A Bayesian decision approach to rainfall thresholds based flood warning, Hydrol. Earth Syst. Sci., 10, 413-426, doi:10.5194/hess-10-413-2006, 2006.

Neary, D. G. and Swift, L. W.: Rainfall thresholds for triggering a debris avalanching event in the southern Appalachian Mountains, Reviews in Engineering Geology, 7, 81-92, 1987.

Overeem, A., Buishand, T. A., and Holleman, I.: Extreme rainfall analysis and estimation of depth-duration-frequency curves using weather radar, Water Resour. Res., 45, W10424, doi:10.1029/2009WR007869, 2009.
Panziera, L., Germann, U., Gabella, M., and Mandapaka, P.: NORA - Nowcasting of Orographic Rainfall by means of Analogues, Q. J. Roy. Meteorol. Soc., 137, 2106-2123, 2011.

Sene, K.: Flash Floods - forecasting and warning, Springer Netherlands, 2013.

Sideris, I. V., Gabella, M., Erdin, R., and Germann, U.: Real-time radar-rain-gauge merging using spatio-temporal co-kriging with external drift in the alpine terrain of Switzerland, Q. J. Roy. Meteorol. Soc., 140, 1097-1111, 2014a.

Sideris, I. V., Gabella, M., Sassi, M., and Germann, U.: The CombiPrecip experience: development and operation of a real-time radar-raingauge combination scheme in Switzerland, 2014b.

Sideris, I. V., Germann, U., Gabella, M., and Panziera, L.: Nowcasting and large-radar-archive statistical learning in Switzerland, in: Proc. 10th Intern. Workshop on Precipitation in Urban Areas, ETH Zürich, Institute of Environmental Engineering, 2015.

Smirnov, N.: Table for estimating the goodness of fit of empirical distributions, Ann. Math. Stat., 19, 279-281, 1948.

Sweeney, T. L.: Modernized Areal Flash Flood Guidance, NOAA Technical Report NWS HYDRO 44, Hydrology Laboratory, National Weather Service, NOAA, Silver Spring, MD, 1992.

Turner, B. J., Zawadzki, I., and Germann, U.: Predictability of Precipitation From Continental Radar Images. Part III: Operational Nowcasting Implementation (MAPLE), J. Appl. Meteorol., 43, 231-248, 2004.

Wieczorek, G. F. and Glade, T.: Climatic factors influencing occurrence of debris flows, in: Debris-flow hazards and related phenomena, edited by: Jakob, M. and Hungr, O., Springer, Berlin Heidelberg, 2005.

Wilson, J. W., Feng, Y., Chen, M., and Roberts, R.: Nowcasting challenges during the Beijing Olympics: successes, failures, and implications for future nowcasting systems, Weather Forecast., 25, 1691-1714, 2010. 